\title{
Motivations intrinsèques et extrinsèques des utilisateurs de systèmes de covoiturage
}

\section{Intrinsic and extrinsic motivations of car sharing systems users}

\author{
Marcos Lima ${ }^{1}$, Patricia Baudier², Karim Alawamleh³ ${ }^{3}$ Bjorn Seguin ${ }^{4}$ \\ ${ }^{1}$ Skema Business School, Université Côte D’Azur, France, marcos.lima@skema.edu \\ 2 École de Management de Normandie, France, pbaudier@em-normandie.fr \\ ${ }^{3}$ MBA in Digital Marketing Strategy, De Vinci Research Center, France, karim.alawamleh@devinci.fr \\ ${ }^{4}$ MBA in Digital Marketing Strategy, De Vinci Research Center, France, bjorn.seguin@devinci.fr
}

RÉSUMÉ. L'économie du partage a fait émerger de nouveaux systèmes produit-service, à la fois respectueux de l'environnement et économiquement attractifs pour toutes les parties-prenantes engagées. Cet article vise à mieux comprendre les motivations des utilisateurs des plateformes de co-voiturage, en étudiant les facteurs intrinsèques et extrinsèques qui expliquent leur attitude et leur comportement d'adoption de ces systèmes. A cet égard, nous avons adapté une échelle basée sur la théorie de l'autodétermination afin d'examiner les motivations de 158 utilisateurs de services de covoiturage. Nous avons constaté que les deux facteurs intrinsèques (le sentiment écologique et le plaisir) et un des facteurs extrinsèques (l'avantage économique) contribuent fortement à créer une attitude favorable en vue de cette pratique. Cependant, les attentes en matière de réputation ne semblent pas influencer les attitudes ou l'intention comportementale de l'utilisateur. Les implications managériales de ces résultats pour l'innovation dans ce domaine seront présentées.

ABSTRACT. The sharing economy enables the emergence of new Product-Service Systems, which are both environmentally friendly and economically attractive business models for all stakeholders involved. This article examines the motivations of users of car sharing platforms, investigating intrinsic and extrinsic drivers behind their attitude and behavior in adopting these systems. To do this, we have adapted a scale based on Self-Determination Theory in order to examine the intentional behavior of 158 car sharing service users. We found that both intrinsic motivation factors (environmental perspective and enjoyment) and one extrinsic factor (economic benefit) strongly contribute to creating a favorable attitude concerning the practice of car sharing. However, expectations surrounding reputation do not seem to influence users' attitudes or behavioral intention. We discuss the managerial implications of these findings for innovation in this domain.

MOTS-CLÉS. Économie du partage, Systèmes Produit-Services, Motivations Intrinsèques et Extrinsèques, Modèles d'Équations Structurelles.

KEYWORDS. sharing economy, Product-Service Systems, intrinsic and extrinsic motivations, structural equation models.

\section{Introduction}

$\mathrm{Au}$ cours des dernières années, les plateformes de technologie de l'information et de la communication (TIC) ont facilité les nouvelles formes de partage, à travers la création d'un marché virtuel où il est possible d'échanger des biens ou des produits « as a service » (« en tant que service », [SCH 15]). Cette économie du partage permet l'émergence de modèles d'innovations durables en créant des solutions à la fois engagées socialement et économiquement attrayantes, fondées sur des relations entre individus (peer-to-peer) de « consommation collaborative » [HAM 15]. Il s'agit donc de modèles d'affaires relativement nouveaux, proposés par Internet, pour contourner ou minimiser le problème de l'asymétrie informationnelle, diminuant les barrières entre l'offre et la demande. Ces modèles ont un certain nombre de caractéristiques communes avec d'autres systèmes de produitservice (SPS) typiques. En éliminant ou réduisant la nécessité de posséder un bien, ces systèmes transforment certains produits (i.e. voitures) en services (i.e. co-voiturage), incitant ainsi les individus à partager leurs ressources existantes. Ainsi faisant, ces réseaux collaboratifs réduisent 
l'empreinte écologique de toutes les parties prenantes impliquées [HEI 13], en intensifiant et en optimisant l'utilisation de ressources existantes. Cet article vise à mieux comprendre les motivations des utilisateurs des plateformes de co-voiturage (PCV), soulignant l'importance des facteurs intrinsèques (comme la durabilité ou bien la jouissance) et extrinsèques (comme la réputation ou bien les avantages économiques). Ces facteurs permettent notamment d'expliquer les comportements des individus en matière d'adoption de ces systèmes.

Le texte est divisé en trois grandes parties : l'introduction, suivie d'une revue de la littérature permettant de conceptualiser les concepts clés (économie du partage et systèmes produit-service) mais aussi d'appréhender les motivations des individus à participer au sein de l'économie du partage. La deuxième partie décrit la méthodologie utilisée et les résultats obtenus. Enfin, en conclusion, nous discutons les implications managériales de ces résultats.

\section{Revue de littérature}

Il n'y a pas de définition universellement acceptée de «l'économie du partage ». Des termes tels que « consommation collaborative », « économie de production par les pairs », « économie peer-topeer » ou " économie de la fonctionnalité » sont souvent utilisés comme synonymes ou équivalents du terme économie du partage [KOO 14, GAL 16]. Quelle que soit la terminologie choisie, tous ces concepts se réfèrent à une relation directe entre le producteur et le consommateur, souvent médiatisée par des plateformes en ligne C2C (Consumer to Consumer) ou B2C (Business to Consumer), permettant de partager des biens et des services entre des individus qui ne se connaissent pas. En d'autres termes, ces plates-formes de TIC créent de nouveaux marchés qui réunissent des individus pour partager ou échanger des produits et des services pour un bénéfice mutuel, monétisé ou non.

Selon Botsman et Rogers [BOT 10], il existe trois grands types d'innovations dans l'économie du partage : (1) les systèmes de services-produits (SPS) ; (2) les marchés de redistribution et (3) les solutions de style de vie collaboratif. Les plateformes de co-voiturages sont principalement positionnées comme une solution SPS centrée sur la performance d'usage [TUK 04, HEI 13, BEL 15]. Ces solutions promettent une utilisation plus efficace des ressources grâce à leur optimisation, favorisant ainsi des modèles économiques plus positifs pour l'environnement [LAP 13]. Dans le secteur des transports, cette catégorie comprend des grandes entreprises de co-voiturage multinationales comme Uber et Grab, mais aussi des services «peer-to-peer » comme Drivy et Ouicar ainsi que des systèmes « pay-per-ride » comme Autolib ou Zipcar [SUN 13].

Les avantages potentiels des SPS de co-voiturage sont multiples [LEB] : diminution des coûts de maintenance, flexibilité accrue quant à l'utilisation du bien en fonction des besoins spécifiques, réduction des impacts environnementaux, mais aussi des gains économiques. En dépit de ces avantages, certains inconvénients sont également mentionnés dans la littérature : perte de la valeur symbolique de la propriété, comportement négligent, conduisant à des actifs dégradés plus rapidement, et coûts de transaction et de livraison supportés par les services logistiques [KAN 08]. En termes d'impacts sociaux, les SPS peuvent, par l'augmentation des ventes et des activités de service, compenser la perte d'emplois dans les industries manufacturières [BAI 07]. Néanmoins, du côté des consommateurs et des producteurs, un changement de culture s'impose: les consommateurs doivent s'adapter à l'idée de posséder moins de produits et les producteurs doivent adapter leur stratégie organisationnelle, leur culture et leurs compétences pour tirer parti de ces nouvelles opportunités.

La montée en puissance de la société en réseau présente de nouvelles opportunités et de nouveaux défis pour les SPS, qui deviennent un pilier de l'économie du partage. Dans les années 1990 et au 
début des années 2000, la plupart des exemples de solutions SPS étaient dans le domaine du « business-to-business » (comme l'approche « solution de bureau » de Xerox, le service «power by the hour » pour les turbines Rolls Royce ou la métrique de maintenance par kilomètre proposée par Michelin pour les flottes de camion). Avant la connectivité Internet à grande échelle, les solutions SPS impliquaient donc rarement l'utilisation des plateformes « peer-to-peer » (même si les services de covoiturage sont cités comme un exemple précoce des principes SPS dans l'un des premiers documents à utiliser le terme - voir [GOE 99].

La disponibilité généralisée de l'Internet sur les smartphones a permis l'émergence de puissants modèles économiques au sein de l'économie du partage à l'échelle mondiale, tel que Airbnb et Uber [ZER 17]. Les vélos et scooters à la demande, ainsi que les solutions de co-voiturage sont maintenant monnaie courante dans les grandes villes du monde entier. Ces plateformes de «business-to-consumers», étant sur des applications, surmontent un bon nombre d'obstacles culturels, comme les incompréhensions au niveau de la langue ou des codes culturels, comme cités auparavant [HAM 15]. Elles offrent des commodités comme la géolocalisation ou bien des systèmes de classements participatifs permettant aux consommateurs de bénéficier des services en toute confiance. Néanmoins, ces nouveaux modes de partage ne sont pas sans critique [MAL 14]. Selon Schor [SCH 16], les acteurs de l'économie du partage obtiennent de la valeur souvent en échappant aux contraintes légales, minimisant le paiement d'impôts et imposant des modes de concurrence perçus comme déloyaux. Le modèle économique de Uber, par exemple, ne serait pas viable avec les prix actuels. L'entreprise perd plusieurs milliards de dollars par an dans l'espoir de pouvoir introduire un jour des voitures autonomes à la place des conducteurs humains actuels. Un tel scénario aurait de conséquences néfastes pour les milliers d'emplois concernés dans le monde entier.

La théorie de l'autodétermination (TAD), développée à l'origine par Ryan et Deci [DEC 71, RYA 00, RYA 17] indique que la motivation basée sur des récompenses extrinsèques (l'argent, un prix) n'est pas suffisante pour conduire à une performance durable, et a donc tendance à disparaître lorsque les récompenses externes sont arrêtées. La motivation intrinsèque (auto-déterminée), au contraire, est le résultat de l'alignement entre les valeurs propres des individus et l'activité ellemême. Du côté du spectre de la motivation, il y a donc des comportements qui ne sont pas autodéterminés, qui sont le résultat de sources externes de récompense et de punition. De l'autre côté, se trouvent les comportements stimulés par la motivation intrinsèque. À mesure que les sources de motivation passent des motifs extrinsèques aux motivations intrinsèques, les individus deviennent plus engagés, intéressés et satisfaits vis-à-vis d'une tâche donnée. Paradoxalement, la présence des facteurs extrinsèques, comme l'argent, peut avoir un impact négatif sur la motivation intrinsèque [PRI 77].

De nombreuses études ont utilisé les facteurs de motivation extrinsèques / intrinsèques pour comprendre l'implication des utilisateurs dans l'économie du partage en général et dans les systèmes produit-service en particulier [DIA 2017]. Belotti et al. [BEL 15], par exemple, ont examiné certaines motivations extrinsèques (telles que l'argent, la réputation et la commodité) et intrinsèques (telles que l'auto-amélioration, la durabilité et l'amusement). Leurs résultats indiquent que, malgré le discours hautain des fournisseurs et des utilisateurs, les avantages économiques sont souvent la principale préoccupation des participants. Cependant, cela n'exclut pas les sentiments authentiques comme l'altruisme et la durabilité [SCH 16].

L'étude de Hamari et al. [HAM 15] s'est intéressée à la motivation à participer à des services de consommation collaborative. Ils ont développé un cadre basé sur la triangulation de trois sources d'inspiration : (a) la théorie de l'auto-détermination (b) des études sur les facteurs motivant le partage économique et (c) des ajustements spécifiques au contexte. Sur la base de ce cadre théorique, ils ont développé un modèle d'équation structurelle qui tient compte des facteurs de 
motivation intrinsèques (durabilité, jouissance) et extrinsèques (réputation, avantages économiques). Dans notre étude, nous avons cherché à adapter leur modèle pour cibler spécifiquement les utilisateurs des plateformes de covoiturage (PCV). Dans les sections ci-dessous, chaque dimension du modèle et les hypothèses correspondantes sont discutées.

\subsection{Durabilité}

Le respect de l'environnement (tout en fidélisant les clients) est l'un des principaux arguments avancés pour justifier l'existence des solutions du type SPS. Dans leur rapport fondateur au gouvernement néerlandais, Goedkoop et al. [GOE 99] positionnent le covoiturage comme un exemple de l'éconologie (c.-à-d., économie + écologie). Les auteurs soutiennent que l'autopartage pourrait entraîner une diminution du nombre de voitures produites, entretenues et procédées et une diminution du besoin de places de stationnement. En outre, comme le soutient Tukker [TUK 04], les SPS de co-voiturage découragent l'utilisation intensive des ressources, car elles fonctionnent selon un système de paiement à la consommation, ce qui stimule l'accès à des solutions plus respectueuses de l'environnement, comme les transports en commun. L'aspect environnemental des PCV est donc considéré comme une motivation intrinsèque selon la théorie de l'autodétermination. Par conséquent, dans le cadre de notre recherche, nous postulons l'hypothèse que le développement durable est perçu comme un facteur intrinsèque prédictif de l'attitude $(\mathrm{H} 1)$ et de l'intention $(\mathrm{H} 2)$ de participer aux PCV. Une attitude est ici définie comme la façon de penser ou de ressentir par rapport au co-voiturage; par «intention », on entend l'objectif explicite d'utiliser un système de covoiturage.

\subsection{Plaisir}

Dans le cadre de l'utilisation de services comme Uber ou Drivy, utiliser différentes voitures selon ses besoins et parfois ne pas avoir besoin de conduire est supposé amener à une expérience plus riche que d'utiliser les transports en commun (moins pratiques) ou d'appeler un taxi [HAW 16]. La communication des SPS de covoiturage met également en évidence le plaisir de se faire conduire plutôt que d'affronter les situations stressantes d'une circulation dense dans les grands centres urbains. Le plaisir est donc supposé être un élément fondamental de la motivation intrinsèque pour l'attitude (H3) et l'intention (H4) d'utilisation des solutions PCV. Dans notre étude, nous avons adopté une sous-échelle, à cinq items, développée par Van der Heijden [VAN 04] pour mesurer l'intérêt suscité par ces solutions et l'acceptation des PCV par ses utilisateurs du point de vue hédonique (voir Tableau A.1 en annexe).

\subsection{Réputation}

Le prestige et l'image de soi semblent être un moteur extrinsèque important dans certaines communautés en ligne [HAR 01]. Participer dans une communauté et avoir une bonne notation par les pairs est sensé augmenter l'estime de soi et combler les besoins de reconnaissance. Ceci est particulièrement observable dans les communautés de développement collaboratif des logiciels en mode « open source » [KAN 05, WAS 05]. Par conséquent, nous supposons que les participants au PCV pourraient s'attendre à obtenir un statut supérieur ou une récompense intangible quand ils participent à une communauté et qu'obtenir une bonne note dans le système de notation de ces plateformes ferait partie des motivations extrinsèques. Selon ce principe, nous postulons que la perspective d'améliorer sa réputation a un impact direct, positif et significatif sur l'attitude (H5) et sur l'intention (H6) de participer à de tels services.

\subsection{Avantage économique}

Il est bien accepté parmi les chercheurs de la théorie de l'autodétermination que les avantages économiques sont une forte motivation extrinsèque [KOO 14, FRA 15, BOC 05). Participer aux 
PCV est, en effet, souvent économiquement plus attractif que de s'acheter une voiture ou même de prendre un taxi. Par conséquent, nous supposons que les avantages économiques sont un facteur extrinsèque important qui influence les attitudes $(\mathrm{H} 7)$ et les intentions (H8) des participants à l'égard des PCV.

\subsection{Attitude et Intention comportementale}

Dans la tradition des études du comportement du consommateur [AJZ 91], la motivation n'est pas toujours directement liée à l'intention d'adopter un service ou d'acheter un produit ; elle est souvent influencée par les attitudes envers l'objet étudié. Pour cette raison, les attitudes envers les PCV doivent donc être considérées comme un facteur d'influence qui peut avoir un impact positif, direct et significatif sur l'intention comportementale des participants (H9) dans notre modèle global. L'intention elle-même peut être mesurée par la sous-échelle de continuité comportementale de Bhattacherjee [BHA 01].

\section{Méthodologie}

Afin de tester nos hypothèses (Tableau 1), nous avons utilisé la méthode des moindres carrés. Basée sur la variance, cette approche permet de mieux comprendre les relations de type cause à effet (HAIR et al., 2012). Le logiciel SmartPLS3 (développé par RINGLE, WENDE, WILL, 2005) a été utilisé pour tester la fiabilité et la validité du modèle interne ainsi que pour analyser le modèle externe.

\begin{tabular}{|c|c|c|}
\hline Durabilité & $\begin{array}{l}\text { La durabilité perçue des PCV a un impact direct, positif et significatif sur } \\
\text { l'Attitude de ses utilisateurs } \\
\text { La durabilité perçue des PCV a un impact direct, positif et significatif sur } \\
\text { l'Intention Comportementale de ses utilisateurs }\end{array}$ & $\mathrm{H} 1$ \\
\hline Plaisir & $\begin{array}{l}\text { Le plaisir perçu de l'utilisation des PCV a un impact direct, positif et } \\
\text { significatif sur l'Attitude de ses utilisateurs } \\
\text { Le plaisir perçu de l'utilisation des PCV a un impact direct, positif et } \\
\text { significatif sur l'Intention Comportementale de ses utilisateurs }\end{array}$ & $\begin{array}{l}\mathrm{H} 3 \\
\mathrm{H} 4\end{array}$ \\
\hline \multirow{2}{*}{ Réputation } & $\begin{array}{l}\text { La perception de la réputation gagnée en participant à la communauté des } \\
\text { PCV a un impact direct, positif et significatif sur l'Attitude de ses utilisateurs }\end{array}$ & H5 \\
\hline & $\begin{array}{l}\text { La perception de la réputation gagnée en participant dans une communauté du } \\
\text { type PCV a un impact direct, positif et significatif sur l'Intention } \\
\text { Comportementale de ses utilisateurs }\end{array}$ & H6 \\
\hline \multirow{2}{*}{$\begin{array}{l}\text { Avantages } \\
\text { économiques }\end{array}$} & $\begin{array}{l}\text { Les avantages économiques perçus dans l'utilisation des PCV ont un impact } \\
\text { direct, positif et significatif sur l'Attitude de ses utilisateurs }\end{array}$ & $\mathrm{H} 7$ \\
\hline & $\begin{array}{l}\text { Les avantages économiques perçus dans l'utilisation des PCV ont un impact } \\
\text { direct, positif et significatif sur l'Intention Comportementale de ses utilisateurs }\end{array}$ & H8 \\
\hline Attitude & $\begin{array}{l}\text { L'attitude des utilisateurs par rapport aux PCV a un impact direct, positif et } \\
\text { significatif sur leur Intention Comportementale }\end{array}$ & H9 \\
\hline
\end{tabular}

Tableau 1. Liste des hypothèses 


\subsection{Données et échantillon}

Une méthode quantitative basée sur le processus CAWI (Computer Assisted Web Interviewing) a été utilisée pour tester notre modèle théorique. Un questionnaire en ligne a été créé via Google Form, les répondants ayant eu le choix de répondre quand ils le souhaitaient sur leur téléphone mobile, leur tablette ou leur ordinateur. Un échantillon de 158 répondants a été collecté par l'intermédiaire d'une enquête en ligne auto-administrée auprès d'utilisateurs de plateformes de covoiturage en utilisant un procédé d'échantillonnage non probabiliste. Le questionnaire, construit à partir d'échelles existantes (Annexe A.1), a été administré auprès d'une population dite digital natives en utilisant la page Facebook de deux étudiants en Master II. Nés après 1980 [PRE 01], ils sont considérés comme les premiers adeptes de nouveaux services basés sur le Web [HOW 00]. De plus, les digital natives sont identifiés comme étant plus préoccupés par le développement durable que la population dite digital immigrants [WIL 11]. Notre échantillon est composé de 52,5\% de répondants ayant moins de 25 ans et $47,5 \%$ compris entre 26 et 37 ans. Les hommes représentaient $56 \%$ de l'échantillon, contre $44 \%$ de femmes.

\section{Résultats empiriques}

\subsection{Validation du modèle de mesure}

Cinq items, dont les saturations étaient en dessous du seuil recommandé de 0,7 , ont été retirés du modèle (Tableau 2).

\begin{tabular}{|c|c|}
\hline Item & \\
\hline ATT3 & $\begin{array}{l}\text { I think participating in car/ride sharing is a good thing. / Je } \\
\text { pense que participer au covoiturage est une bonne chose. }\end{array}$ \\
\hline ATT5 & $\begin{array}{l}\text { Car/ride sharing is a better mode of consumption than using a } \\
\text { private car. / Le partage de voiture est un meilleur mode de } \\
\text { consommation que d'utiliser une voiture privée. }\end{array}$ \\
\hline ENJ4 & $\begin{array}{l}\text { I think car/ride sharing is interesting. / Je pense que le } \\
\text { covoiturage est intéressant. }\end{array}$ \\
\hline EXTR4 & $\begin{array}{l}\text { My participation in car/ride sharing saves me time. / Ma } \\
\text { participation au partage de voiture / trajet me fait gagner du } \\
\text { temps. }\end{array}$ \\
\hline SUST2 & $\begin{array}{l}\text { Car/ride sharing is a sustainable mode of consumption. / Le } \\
\text { covoiturage est un mode de consommation durable. }\end{array}$ \\
\hline
\end{tabular}

Tableau 2. Items supprimés du modèle

La validité convergente (AVE>0,5) (Tableau A.2) ainsi que la validité discriminante (Tableau A.3) ont été contrôlées. Les indices de concordance et les valeurs de l'alpha de Cronbach sont supérieurs à 0,7 (Tableau A.2). Par conséquent, la fiabilité et la validité du modèle externe sont confirmées.

\subsection{Test du modèle structurel}

Le modèle interne a été estimé en analysant pour chaque variable les $R^{2}, f^{2}$ et $Q^{2}$. Le $R^{2}$ exprime la proportion de la variance expliquée par les variables endogènes. Selon Chin (1998), le $\mathrm{R}^{2}$ est considéré comme substantiel $(0,7)$, modéré $(0,33)$ ou faible $(0,19)$. L'effet de taille sur une variable 
endogène est analysé en étudiant le $\mathrm{f}^{2}$. Un $\mathrm{f}^{2}$ à 0,02 indique un faible effet sur le $\mathrm{R}^{2}$; un effet est considéré comme modéré à 0,15 et élevé à 0,35 [COH 98]. La fiabilité de la prédiction du modèle est validé en vérifiant que les $\mathrm{Q}^{2}$ sont tous supérieurs à 0 . Les relations entre les variables ont été contrôlées en vérifiant que les paramètres étaient supérieurs à 0,200 , les valeurs $t$ supérieures à 1,96 et les valeurs p inférieures à 0,05 . L'indice de qualité globale (Goodness of Fit) à 0,49 confirme la haute qualité du modèle [LAT 12].

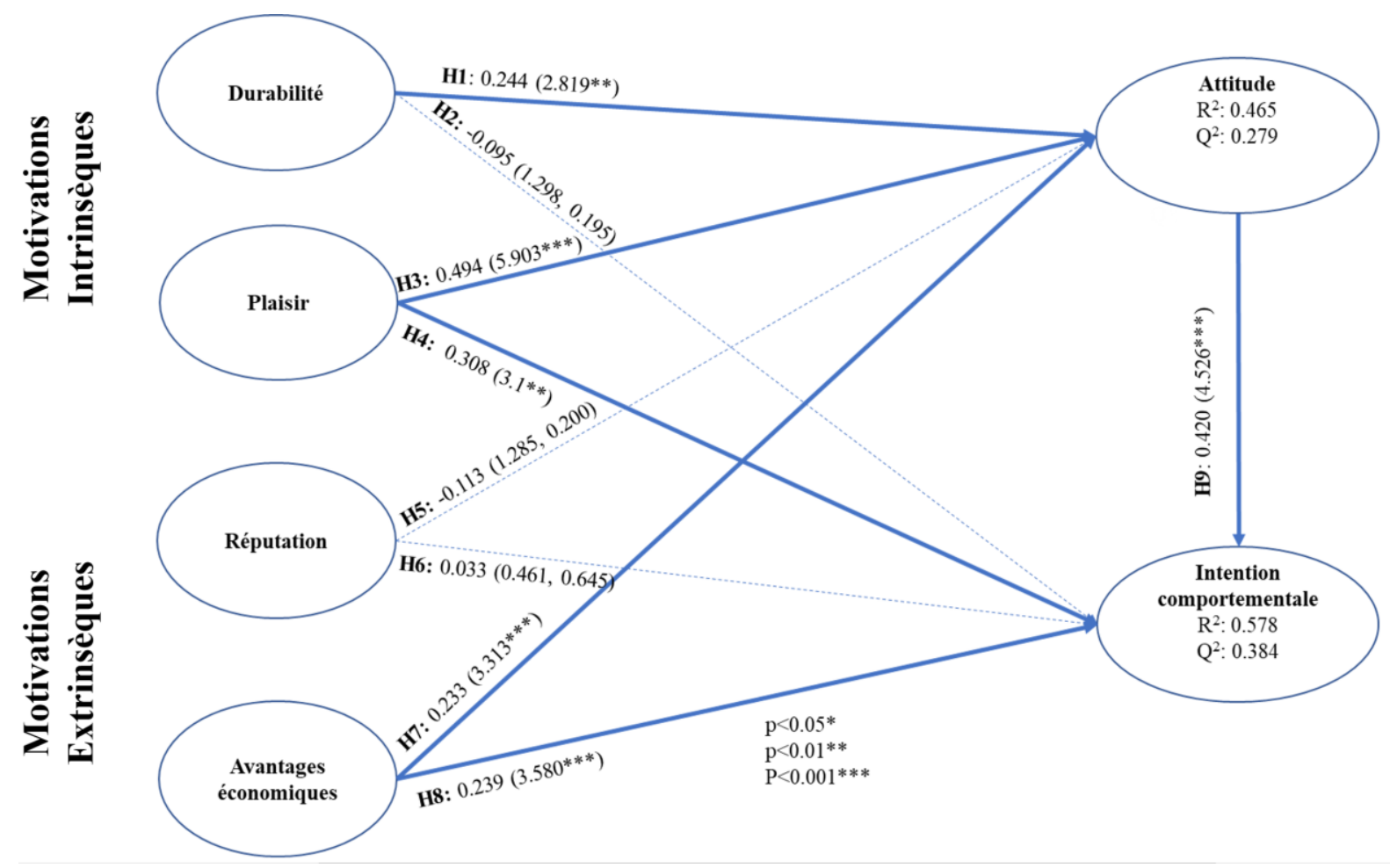

Figure 1. Modèle hypothétique (hypothèses validées en gras)

\subsection{Test des hypothèses}

\subsubsection{Attitude}

La valeur $\mathrm{R}^{2}$ du construit Attitude à 0,465 est considérée comme correcte. Le test de prédictivité $\mathrm{Q}^{2}$ à 0,279 démontre une bonne pertinence prédictive. L'effet de taille $\left(\mathrm{f}^{2}\right) \mathrm{du}$ plaisir sur l'attitude est considéré comme élevé $(0,287)$; l'effet de la taille de la réputation est considéré comme faible $(0,014)$ et le $\mathrm{f}^{2}$ des avantages économiques $(0,085)$ et durabilité $(0,080)$ sont modérés.

Hypothèse H1 : Le paramètre (Path coefficient) de 0,244, la valeur $t$ de 2,819 et la valeur $p$ de 0,005 confirment que l'impact de la durabilité sur l'attitude est positif, direct et significatif. Par conséquent, l'hypothèse $\mathrm{H} 1$ est validée.

Hypothèse H3 : Le plaisir a un impact positif (coefficient de 0,494) et significatif (valeur de $\mathrm{t}=$ 5,903, valeur de $p=0,000$ ) sur l'attitude. L'hypothèse H3 est donc supportée.

Hypothèse $\mathbf{H 5}$ : La réputation n'a pas d'impact sur Attitude (coefficient $-0,133, \mathrm{t}=1,285, \mathrm{p}=$ 0,2). H3 n’est pas supportée.

Hypothèse $\mathbf{H 7}$ : Les avantages économiques ont un effet direct et positif sur Attitude (coefficient $0,233, \mathrm{t}=3,313, \mathrm{p}=0,001)$. Par conséquent, $\mathrm{H} 7$ est validée. 


\subsubsection{Intention comportementale}

La valeur $\mathrm{R}^{2}$ de la variable d'intention comportementale à 0,578 est considérée comme significative. Les $\mathrm{Q}^{2}$ montrent une pertinence prédictive satisfaisante $(0,384)$. L'effet de taille de l'attitude sur l'intention comportementale est élevé $(0,223)$, le $\mathrm{f}^{2}$ des avantages économiques $(0,105)$ et du plaisir $(0,110)$ sont considérés comme modérés et la réputation $(0,001)$ et la durabilité $(0,014)$ comme faibles.

Hypothèse H2 : La durabilité n'a pas d'impact sur l'intention comportementale (Paramètre = $0,095, t=1,298 ; p=0,195)$. Par conséquent, l'hypothèse H2 n'est pas supportée.

Hypothèse H4 : Le plaisir influence directement et positivement l'intention comportementale avec un paramètre à 0,308 . Cette relation est considérée comme significative (valeur $\mathrm{t}=3,1$, valeur $\mathrm{p}=0,002$ ). Par conséquent, l'hypothèse H4 est validée.

Hypothèse H6 : La réputation n'influence pas l'intention comportementale (Paramètre $=0,033 ; \mathrm{t}$ $=0,461 ; p=0,645)$. Par conséquent, H6 n'est pas validée.

Hypothèse H8 : Avec un paramètre à 0,239, une valeur t de 3,580 et une valeur $\mathrm{p}$ de 0,000 , la variable avantages économiques a un impact direct, positif et significatif sur l'intention comportementale. L'hypothèse H8 est supportée.

Hypothèse $\mathbf{H 9}$ : Attitude avec un paramètre à 0,420, des valeurs $t=4,526$ et $p=0,000$ impacte positivement, directement et significativement le construit Intention comportementale. L'hypothèse H9 est validée.

En résumé, 6 hypothèses ont été validées et 3 rejetées (Figure 1).

L'étude confirme l'influence de la dimension écologique (la préservation de l'environnement), du plaisir et des potentiels avantages économiques sur l'attitude des utilisateurs à utiliser les PCV. L'importance de la dimension du plaisir, du fait de son poids sur la variable, ne doit pas être négligée, les répondants souhaitant mettre en avant l'aspect hédonique de ce type de service. Les personnes peuvent ressentir du plaisir en partageant un véhicule avec d'autres personnes pour échanger, passer un moment agréable.

On constate que l'intention comportementale est impactée par trois construits : l'attitude, le plaisir et les avantages économiques. Notre étude confirme l'importance de l'attitude sur l'intention des utilisateurs à continuer d'utiliser les plateformes de co-voiturage. Ils estiment qu'utiliser le covoiturage est une bonne idée qui fait sens. Toutefois, le plaisir éprouvé lors de l'utilisation de ces services impacte, aussi de façon non négligeable, l'attitude. Il en va de même de l'aspect économique, les personnes souhaitent réaliser des économies soit en utilisant le véhicule d'une autre personne ou bien en proposant de conduire des individus au sein de leur propre véhicule.

Enfin, il s'avère que les individus n'utilisent pas le co-voiturage afin de donner une bonne image d'eux puisque les relations entre la variable réputation et les deux construits mesurés dans le modèle ont été rejetées. Les trois éléments de l'échelle de Hamari et al. [HAM 15] mesurant cette variable étaient les suivants : «a) Contribuer à ma communauté de covoiturage améliore mon image au sein de la communauté ; b) Je gagne de la reconnaissance en contribuant à ma communauté de voiture / covoiturage et c) Je gagnerai le respect des autres en utilisant des services de co-voiturage » (voir Tableau A1 en annexe). Cette approche de la réputation personnelle peut être plus pertinente dans certaines plateformes de partage «peer-to-peer» ou dans les grandes communautés de développement de logiciel en open source, comme discuté précédemment [YAN 10]. 
Pour finir, nous n'avons trouvé aucun effet médiateur de la variable d'attitude sur la relation de durabilité, du plaisir, de la réputation et des avantages économiques sur l'intention comportementale.

\section{Conclusions, implications et recommandations}

Comme prévu, les facteurs de motivation intrinsèques (perceptions de durabilité et de plaisir) contribuent fortement à créer une attitude favorable concernant la pratique du covoiturage. Les avantages économiques sont aussi une source de motivation extrinsèque forte à la fois pour l'Attitude et pour l'Intention Comportementale. Toutefois, les attentes concernant la réputation ne semblent pas influer sur les attitudes de l'utilisateur ou son intention. Cela peut sembler contreintuitif si l'on considère le rôle de la confiance et de l' «e-réputation » dans la collaboration et le partage. Néanmoins, il faut souligner que le modèle ne traitait pas la réputation comme une variable de la confiance mutuelle ou de la confiance réciproque, mais de la façon dont leur participation aux PCV renforcerait leur réputation dans la communauté.

Il est intéressant de noter également que les perceptions quant à la durabilité de la participation au PCV n'expliquent pas directement l'intention comportementale à participer à ces services. Nos résultats sont cohérents avec l'étude de Hamari et al. [HAM 15]. Les auteurs proposent, néanmoins, que l'intention comportementale puisse être indirectement affectée par des attitudes positives découlant des attentes de durabilité. Contrairement aux résultats obtenus par ladite étude, cependant, nous avons trouvé un lien direct entre les avantages économiques et l'attitude vers les PCV. Cela peut suggérer une influence globale plus forte des facteurs de motivation extrinsèques dans le cas des utilisateurs des SPS du type co-voiturage.

\subsection{Implications et recommandations managériales}

Selon notre étude, les facteurs intrinsèques semblent contribuer plus à motiver les utilisateurs de PCV que les facteurs extrinsèques. Ceci est en accord avec la théorie de l'autodétermination. En tenant compte de ce résultat, les PCV devraient positionner leur service comme écologique et hédoniste. Concernant le premier attribut, le problème croissant de la pollution peut être abordé avec une prime des SPS de covoiturage pour privilégier les voitures hybrides ou électriques. En plus, des modes de partage de trajet (comme Uber Pool) doivent rester attractifs du point de vue de leur tarification, de façon à stimuler les utilisateurs à privilégier cette modalité. Les plateformes doivent communiquer sur ces actions pour renforcer leur positionnement écologique. Concernant l'aspect hédoniste de l'utilisation des SPS de covoiturage, les plateformes mettent déjà en avant dans leur communication les avantages liés à la flexibilité et à la tranquillité de leurs services.

L'impact des facteurs économiques est non négligeable. Maintenir les prix des services en dessous des autres solutions équivalentes (taxi, voiture louée) semble essentiel comme facteur de différentiation des PCV. Néanmoins, comme vu dans le cas d'Uber, l'actuelle structure de coûts de certains SPS dans ce domaine ne sont pas soutenables à moyen terme. Trouver d'autres sources de revenu et/ou baisser les coûts devient donc primordial. Les acteurs peuvent poursuivre des pistes palliatives d'augmentation de recettes à court terme (comme par exemple l'insertion de publicités ciblées dans les applications des PCV sur les smartphones et l'utilisation de la surface des voitures pour des campagnes publicitaires). A moyen et long terme, cependant, l'introduction de voitures autonomes semble incontournable pour rendre leurs business models économiquement viables [SCH 17, BEL 17).

Plusieurs difficultés s'imposent à l'élimination des conducteurs « humains » [LOZ 12] : du point de vue légal, la réglementation des voitures autonomes et toutes les questions éthiques liées à la 
responsabilité civile en cas d'accident risquent de prendre du temps. D'un point de vue technologique, ces innovations demandent un investissement colossal en recherche et développement. Ce n'est pas étonnant que toutes les grandes entreprises du secteur automobile se soient lancées dans cette course à l'innovation. Enfin, d'un point de vue socio-économique, le chômage massif provoqué par cette innovation radicale pourrait avoir un fort impact sociétal, ce qui peut amener les gouvernements à retarder l'autorisation de ce mode opératoire [LIT 14]. Ensemble, ces trois tendances posent des barrières considérables à la viabilité des PCV dans le format actuel. Cela pourrait représenter une opportunité pour les plateformes de location de voitures de particulierà-particulier, qui ont moins de contraintes légales, technologiques et socio-économiques.

Enfin, le dernier résultat de notre étude concerne l'influence nulle de la réputation dans l'attitude et le comportement des usagers. Tel qu'il est conçu à ce jour, le système de réputation et de notation des voyageurs ne semble pas motiver les utilisateurs. Les PCV peuvent développer des systèmes beaucoup plus performants à partir des principes de «gamification» (i.e. rendre ludique l'expérience d'utilisation d'un service, voir par exemple [ZEP 16]. Compte tenu de la grande importance attribuée par les usagers à l'aspect environnemental, les entreprises de co-voiturage peuvent établir un «score » d'empreinte écologique lié au type des véhicules (hybride, électrique ou à carburant) et au type de covoiturage (partagé ou seul). Le partage de ce type de résultat écologique parmi les utilisateurs peut à la fois stimuler l'utilisation des services et augmenter la motivation intrinsèque des participants.

Notre travail comprend certaines limitations : nos conclusions exploratoires sont basées sur un échantillon non représentatif et de convenance (non probabiliste). Malgré la petite taille de cet échantillon, nous avons démontré la robustesse des données et la consistance du modèle d'équations structurelles. L'échantillon des répondants est principalement composé de digital natives (nés après 1981) et les résultats sont donc plus intimement liés aux attitudes et comportements de cette génération. Il serait intéressant de comparer cette étude avec des résultats concernant la génération $\mathrm{X}$ (nés entre les années 1960 et 1980) ou les baby-boomers (1940-1960).

Concernant l'échelle, nous recommandons à de futurs chercheurs de changer les questions relatives à la réputation proposées par l'échelle de Hamari et al. [HAM 15]. La formulation des items s'est avérée peu pertinente afin d'expliquer les motivations des usagers. A leur place, on pourrait poser des questions qui mettent en évidence les aspects liés à la confiance dans les systèmes de co-voiturage. En effet, si les utilisateurs sont peu soucieux de la note attribuée par les chauffeurs, la réputation des chauffeurs établie par les usagers semble être un critère important de choix d'un service de co-voiturage. Cette hypothèse doit être testée par des études confirmatoires.

Cette étude a eu pour objectif de mieux comprendre les motivations intrinsèques et extrinsèques des utilisateurs des systèmes produit-service dans le domaine du partage de voitures. Nos résultats confirment l'importance des facteurs intrinsèques (comme la perception de durabilité et l'hédonisme) mis en évidence par la théorie de l'autodétermination, ainsi que l'importance du facteur extrinsèque lié à l'économie pécuniaire. Les résultats permettent de relativiser le rôle de la réputation comme facteur motivationnel. Les implications managériales discutées peuvent servir de base à une stratégie marketing et d'innovation dans les modèles économiques des entreprises de covoiturage mais aussi pour leurs concurrents indirects, comme les entreprises de location de voiture entre individus.

\section{Bibliographie}

[AJZ 91] AJZEN, I. (1991). «The theory of planned behavior. » Organizational behavior and human decision processes, 50(2), 179-211. 
[bai 07] Baines, T. S., Lightfoot, H. W., Evans, S., Neely, A., Greenough, R., Peppard, J., \& Alcock, J. R. (2007). « State-of-the-art in product-service systems. » Journal of engineering manufacture, 221(10), 1543-1552.

[Bel 17] Bellos, I., Ferguson, M., \& TOKTAY, L. B. (2017). « The car sharing economy: Interaction of business model choice and product line design. » Manufacturing \& Service Operations Management, 19(2), 185-201.

[bel 15] Bellotti, V., Ambard, A., Turner, D., Gossmann, C., Demkova, K., \& Carroll, J. M. (2015). «A muddle of models of motivation for using peer-to-peer economy systems. » In Proceedings of the 33rd Annual ACM Conference on Human Factors in Computing Systems (pp. 1085-1094). ACM.

[BHA 01] BHATTACHERJEE, A. (2001). «Understanding information systems continuance: an expectation-confirmation model. » MIS quarterly, 351-370.

[BOt 10] Botsman, R., \& Rogers, R. (2010). «Beyond zipcar: Collaborative consumption. » Harvard Business Review, 88(10), 30.

[вос 05] Bоск, G. W., ZMUd, R. W., KiM, Y. G., \& LEe, J. N. (2005). « Behavioral intention formation in knowledge sharing: Examining the roles of extrinsic motivators, socialpsychological forces, and organizational climate. »MIS quarterly, 87-111.

[CHI 98] CHIN, W. (1998), « Issues and opinion on structure equation modeling. » MIS Quarterly, 22(1), 7-16.

[COH 88] COHEN, J. (1988), Statistical Power Analysis for the Behavioral Sciences, Second edition, Lawrence Erlbaum Associates, INC.

[Dia 17] Dias, F. F., Lavieri, P. S., Garikapati, V. M., Astroza, S., Pendyala, R. M., \& Bhat, C. R. (2017). «A Behavioral Choice Model of the Use of Car-Sharing and Ride-Sourcing Services. » Transportation, 44(6), 13071323. (No. 17-06359).

[DEC 71] DECI, E. L. (1971). «Effects of externally mediated rewards on intrinsic motivation. » Journal of Personality and Social Psychology, 18, 105-115.

[Fra 15] Fraiberger, S. P., \& Sundararajan, A. (2015). Peer-to-Peer Rental Markets in the Sharing Economy (No. 15-19).

[Gal 16] Gallaud, D., \& LAPERChe, B. (2016). Circular Economy, Industrial Ecology and Short Supply Chain: Towards Sustainable Territories. John Wiley \& Sons.

[gOe 99] Goedkoop, M., Van Haler, C., Te Riele, H., and Rommers, P. (1999) «Product Service-Systems, ecological and economic basics. » Report for Dutch Ministries of Environment (VROM) and Economic Affairs (EZ).

[HAi 12] Hair, J., SARStedt, M., Ringle, C., Mena, J. (2012), «An assessment of the use of partial least squares structural equation modeling in marketing research. » Journal of the Academic Marketing Science, 40(3), 414-433.

[HeI 13] HeInRICHS, H. (2013). Sharing Economy: A Potential New Pathway to Sustainability. Gaia, 22(4), 228-231.

[HAM 15] HAMARI, J., SJÖKLINT, M., \& UKKONEN, A. (2015). "The sharing economy: Why people participate in collaborative consumption. » Journal of the Association for Information Science and Technology, 67(9), 20472059.

[HAR 01] HARS, A., \& OU, S. (2001, January). «Working for free? Motivations of participating in open source projects. » In System Sciences, 2001. Proceedings of the 34th Annual Hawaii International Conference on (pp. 9pp). IEEE.

[HAW 16] HAWLiTSCHEK, F., TEUBNER, T., \& GiMPEL, H. (2016, January). « Understanding the Sharing Economy-Drivers and Impediments for Participation in Peer-to-Peer Rental. » In System Sciences (HICSS), 2016 49th Hawaii International Conference on (pp. 4782-4791). IEEE.

[HOW 00] HOWE.N, STRAUSS.W. (2000), Millennials rising: The next great generation. New York, NY: Vintage Books.

[KAN 05] KanKanhalli, A., TAN, B. C., \& WeI, K. K. (2005). "Contributing knowledge to electronic knowledge repositories: an empirical investigation. » MIS quarterly, 113-143.

[KAN 08] KANG, M. J., \& WIMMER, R. (2008). « Product service systems as systemic cures for obese consumption and production. Journal of Cleaner Production, 16(11), 1146-1152. 
[Koo 14] Koopman, C., Mitchell, M., \& Thierer, A. (2014). «The sharing economy and consumer protection regulation: The case for policy change. » J. Bus. Entrepreneurship \& L., 502-529.

[LOZ 12] LOZANO-PEREZ, T. (2012). Autonomous robot vehicles. Springer Science \& Business Media.

[lat 12] Latan, H., GHOZALI, I. (2012) « Partial Least Squares: Concept, Technique and Application using Program SmartPLS for Empirical Research. », BP UNDIP.

[LAP 13] LAPERCHE, B., \& PICARD, F. (2013). « Environmental constraints, Product-Service Systems development and impacts on innovation management: learning from manufacturing firms in the French context. » Journal of Cleaner Production, 53, 118-128.

[LEB 08] LEBEL, L., \& LOREK, S. (2008). « Enabling sustainable production-consumption systems. » Annual Review of Environment and Resources, 33, 241-275.

[LIT 14] LitMAn, T. (2014). «Autonomous vehicle implementation predictions. » Victoria Transport Policy Institute, 28.

[MAL 14] MALhotra, A., \& VAN Alstyne, M. (2014). « The dark side of the sharing economy... and how to lighten it. » Communications of the ACM, 57(11), 24-27.

[PRE 01] PRENSKY, M. (2001), « Digital natives, digital immigrants. » Horizon, 9(5), (2001),1-6.

[PRI 77] PRITCHARD, R.; CAMPBEll, K.; CAMPBELl, D. (1977). « Effects of extrinsic financial rewards on intrinsic motivation. » Journal of Applied Psychology. 62: 9-15

[RIN 05] Ringle, C., Wende, S., \& WILL, A. (2005). Smart-PLS Version 2.0 M3. University of Hamburg.

[RYA 00] RYAN, R. M., \& DECI, E. L. (2000). «Self-determination theory and the facilitation of intrinsic motivation, social development, and well-being. » American psychologist, 55(1), 68.

[RYA 17] RYAN, R. M. \& DECI, E. L. (2017). «Self-determination theory: Basic psychological needs in motivation, development, and wellness. » New York: Guilford Publishing.

[SCH 16] SCHOR, J. (2016). «Debating The Sharing Economy. » Journal of Self-Governance \& Management Economics, 4(3).

[SCH 15] SCHOR, J. B., \& FitZMAURICE, C. J. (2015). "Collaborating and connecting: the emergence of the sharing economy. » Handbook of research on sustainable consumption, 410.

[SUN 13] SUNDARARAJAN, A. (2013). «From Zipcar to the sharing economy. » Harvard Business Review, 1.

[SCH 17] SCHWIETERMAN, J. P., \& PELON, M. (2017). «First Zipcar, Now Uber: Legal and Policy Issues Facing the Expanding 'Shared Mobility' Sector in US Cities. » Belmont L. Rev., 4, 109.

[TUK 04] TUKKER, A. (2004). «Eight types of product-service systems: eight ways to sustainability? Experiences from SusProNet. » Business strategy and the environment, 13(4), 246-260.

[WAS 05] WASKo, M. M., \& FARAJ, S. (2005). «Why should I share? Examining social capital and knowledge contribution in electronic networks of practice. » MIS quarterly, 35-57.

[WIL 11] Williams.K.C., Page.R.A. (2011), «Marketing to the generations. » Journal of Behavioral Studies in Business, 5(1), 1-17.

[VAN 04] VAN DER HEIJDEN, H. (2004). « User acceptance of hedonic information systems. » MIS quarterly, 695-704.

[YAN 10] YANG, H. L., \& LAI, C. Y. (2010). « Motivations of Wikipedia content contributors. » Computers in human behavior, 26(6), 1377-1383.

[ZEP 16] ZEPEDA, R. (2016) «Why Gamification Matters to the Auto Industry. » Linkedin, 9 Mar. 2016, www.linkedin.com/pulse/why-gamification-matters-auto-industry-robert-zepeda/.

[Zer 17] Zervas, G., Proserpio, D., \& BYers, J. W. (2017). « The rise of the sharing economy: Estimating the impact of Airbnb on the hotel industry. » Journal of Marketing Research, 54(5), 687-705. 


\section{Annexes}

Tableau A.1 : Échelles de mesure du questionnaire

\begin{tabular}{|c|c|c|}
\hline Item & & Adapted from / Source \\
\hline ATT1 & $\begin{array}{l}\text { All things considered, I find participating in car/ride } \\
\text { sharing to be a wise move. / Tout bien considéré, je } \\
\text { trouve que participer au covoiturage est judicieux. }\end{array}$ & \multirow[t]{5}{*}{ AJZEN (1991) } \\
\hline ATT2 & $\begin{array}{l}\text { All things considered, I think car/ride sharing is a } \\
\text { positive thing. / Tout bien considéré, je pense que le } \\
\text { covoiturage est une chose positive. }\end{array}$ & \\
\hline ATT3 & $\begin{array}{l}\text { I think participating in car/ride sharing is a good thing. I } \\
\text { Je pense que participer au covoiturage est une bonne } \\
\text { chose. }\end{array}$ & \\
\hline ATT4 & $\begin{array}{l}\text { Overall, sharing a car/ride within a community makes } \\
\text { sense. / Globalement, partager une voiture au sein d'une } \\
\text { communauté a du sens. }\end{array}$ & \\
\hline ATT5 & $\begin{array}{l}\text { Car/ride sharing is a better mode of consumption than } \\
\text { using a private car. / Le partage de voiture est un } \\
\text { meilleur mode de consommation que d'utiliser une } \\
\text { voiture privée. }\end{array}$ & \\
\hline BI1 & $\begin{array}{l}\text { I expect to continue car/ride sharing often in the future. / } \\
\text { Je prévois de continuer à faire du covoiturage souvent. }\end{array}$ & \multirow[t]{4}{*}{$\begin{array}{l}\text { BHATTACHERJEE } \\
(2001)\end{array}$} \\
\hline BI2 & $\begin{array}{l}\text { I can see myself engaging in car/ride sharing more } \\
\text { frequently in the future. / Je compte, dans le futur, } \\
\text { m'engager plus souvent dans le covoiturage. }\end{array}$ & \\
\hline BI3 & $\begin{array}{l}\text { I can see myself increasing my car/ride sharing activities } \\
\text { if possible. / Je compte, si possible, augmenter mes } \\
\text { activités de covoiturage. }\end{array}$ & \\
\hline BI4 & $\begin{array}{l}\text { It is likely that I will frequently participate in car/ride } \\
\text { sharing communities in the future. / Il est probable que } \\
\text { je participerai fréquemment au sein des communautés de } \\
\text { covoiturage à l'avenir. }\end{array}$ & \\
\hline ENJ1 & $\begin{array}{l}\text { I think car/ride sharing is enjoyable. / Je pense que } \\
\text { pratiquer le covoiturage est agréable. }\end{array}$ & \multirow[t]{5}{*}{$\begin{array}{l}\text { VAN DER HEIJDEN } \\
(2004)\end{array}$} \\
\hline ENJ2 & $\begin{array}{l}\text { I think car/ride sharing is exciting. / Je pense que } \\
\text { pratiquer le covoiturage est passionnant. }\end{array}$ & \\
\hline ENJ3 & $\begin{array}{l}\text { I think car/ride sharing is fun. / Je pense que pratiquer le } \\
\text { covoiturage est un plaisir. }\end{array}$ & \\
\hline ENJ4 & $\begin{array}{l}\text { I think car/ride sharing is interesting. / Je pense que } \\
\text { pratiquer le covoiturage est intéressant. }\end{array}$ & \\
\hline ENJ5 & $\begin{array}{l}\text { I think car/ride sharing is pleasant. / Je pense que } \\
\text { pratiquer le covoiturage est plaisant. }\end{array}$ & \\
\hline
\end{tabular}




\begin{tabular}{|c|c|c|}
\hline EXTR1 & $\begin{array}{l}\text { I can save money if I participate in car/ride sharing. / Je } \\
\text { peux économiser de l'argent si je participe au } \\
\text { covoiturage. }\end{array}$ & \multirow{4}{*}{$\begin{array}{l}\text { BOCK et al. }(2005) \text {; } \\
\text { KOOPMAN } \text { et al. } \\
\text { (2014); } \\
\text { FRAIBERGER, } \\
\text { SUNDARAJAN (2015) }\end{array}$} \\
\hline EXTR2 & $\begin{array}{l}\text { My participation in car/ride sharing benefits me } \\
\text { financially. / Ma participation au covoiturage me } \\
\text { procure des avantages financiers. }\end{array}$ & \\
\hline EXTR3 & $\begin{array}{l}\text { My participation in car/ride sharing can improve my } \\
\text { economic situation. / Ma participation au covoiturage } \\
\text { peut améliorer ma situation économique. }\end{array}$ & \\
\hline EXTR4 & $\begin{array}{l}\text { My participation in car/ride sharing saves me time. / Ma } \\
\text { participation au covoiturage me fait gagner du temps. }\end{array}$ & \\
\hline REP1 & $\begin{array}{l}\text { Contributing to my car/ride sharing community improves } \\
\text { my image within the community. / Participer au sein de } \\
\text { la communauté de covoiturage améliore mon image } \\
\text { auprès de cette communauté. }\end{array}$ & \multirow[t]{4}{*}{$\begin{array}{l}\text { KANKANHALLI } \text { et al. } \\
(2005) \text {; WASKO, } \\
\text { FARAJ (2005) }\end{array}$} \\
\hline REP2 & $\begin{array}{l}\text { I gain recognition from contributing to my car/ride } \\
\text { sharing community. / Je gagne de la reconnaissance en } \\
\text { contribuant au sein de ma communauté de covoiturage. }\end{array}$ & \\
\hline REP3 & $\begin{array}{l}\text { I would earn respect from others by sharing with other } \\
\text { people in my car/ride sharing community. / Je gagnerai } \\
\text { du respect auprès des autres personnes en participant au } \\
\text { sein de ma communauté de covoiturage. }\end{array}$ & \\
\hline REP4 & $\begin{array}{l}\text { People in the community who contribute to } \\
\text { car/ridesharing have more prestige than those who do } \\
\text { not. / Les membres de la communauté qui contribuent au } \\
\text { covoiturage ont plus de prestige que ceux qui ne le font } \\
\text { pas. }\end{array}$ & \\
\hline SUST1 & $\begin{array}{l}\text { Car/ride sharing helps save natural resources. / Le } \\
\text { covoiturage aide à économiser les ressources naturelles. }\end{array}$ & \multirow{5}{*}{$\begin{array}{l}\text { HAMARI et al. }(2015) \text {; } \\
\text { TUKKER (2004); } \\
\text { GOEDKOOP et al. } \\
\text { (1999) }\end{array}$} \\
\hline SUST2 & $\begin{array}{l}\text { Car/ride sharing is a sustainable mode of consumption. / } \\
\text { Le covoiturage est un mode de consommation durable. }\end{array}$ & \\
\hline SUST3 & $\begin{array}{l}\text { Car/ride sharing is ecological. / Le covoiturage est } \\
\text { écologique. }\end{array}$ & \\
\hline SUST4 & $\begin{array}{l}\text { Car/ride sharing is efficient in terms of using energy. / } \\
\text { Le covoiturage est efficace en termes d'utilisation } \\
\text { d'énergie. }\end{array}$ & \\
\hline SUST5 & $\begin{array}{l}\text { Car/ride sharing is environmentally friendly. / Le } \\
\text { covoiturage est respectueux de l'environnement. }\end{array}$ & \\
\hline
\end{tabular}


Tableau A.2 Validité Convergente et Fiabilité

\begin{tabular}{|c|c|c|c|c|}
\hline & $\begin{array}{c}\text { Alpha de } \\
\text { Cronbach }\end{array}$ & rho_A & $\begin{array}{c}\text { Fiabilité } \\
\text { composite }\end{array}$ & $\begin{array}{c}\text { Average Variance } \\
\text { Extracted (A VE) }\end{array}$ \\
\hline Attitude & 0,755 & 0,755 & 0,860 & 0,671 \\
\hline $\begin{array}{c}\text { Intention } \\
\text { comportementale }\end{array}$ & 0,873 & 0,879 & 0,913 & 0,726 \\
\hline $\begin{array}{c}\text { Avantages économiques } \\
\text { Plaisir }\end{array}$ & 0,698 & 0,712 & 0,831 & 0,621 \\
\hline Réputation & 0,863 & 0,880 & 0,906 & 0,707 \\
\hline Durabilité & 0,805 & 0,817 & 0,872 & 0,631 \\
\hline
\end{tabular}

Tableau A.3 Validité Discriminante (critère de Fornell-Larcker)

\begin{tabular}{|c|c|c|c|c|c|c|}
\hline & Attitude & $\begin{array}{c}\text { Intention } \\
\text { comportementale }\end{array}$ & $\begin{array}{c}\text { Avantages } \\
\text { économiques }\end{array}$ & Plaisir & Réputation & Durabilité \\
\hline Attitude & 0,819 & & & & & \\
\hline $\begin{array}{c}\text { Intention } \\
\text { comportementale }\end{array}$ & 0,677 & 0,852 & & & & \\
\hline $\begin{array}{c}\text { Avantages } \\
\text { économiques }\end{array}$ & 0,434 & 0,499 & 0,788 & & & \\
\hline Plaisir & 0,596 & 0,617 & 0,317 & 0,841 & & \\
\hline Réputation & 0,369 & 0,397 & 0,310 & 0,588 & 0,795 & \\
\hline Durabilité & 0,456 & 0,311 & 0,330 & 0,387 & 0,488 & 0,822 \\
\hline
\end{tabular}




\begin{tabular}{|c|c|c|c|c|c|c|}
\hline & Attitude & $\begin{array}{c}\text { Intention } \\
\text { comportementale }\end{array}$ & Plaisir & $\begin{array}{c}\text { Avantages } \\
\text { économiques }\end{array}$ & Réputation & Durabilité \\
\hline ATT1 & 0,812 & 0,533 & 0,479 & 0,452 & 0,326 & 0,364 \\
\hline ATT2 & 0,827 & 0,553 & 0,477 & 0,360 & 0,258 & 0,393 \\
\hline ATT4 & 0,819 & 0,577 & 0,510 & 0,255 & 0,324 & 0,365 \\
\hline BI1 & 0,652 & 0,890 & 0,533 & 0,408 & 0,320 & 0,238 \\
\hline BI2 & 0,512 & 0,834 & 0,474 & 0,446 & 0,353 & 0,225 \\
\hline BI3 & 0,534 & 0,798 & 0,518 & 0,402 & 0,353 & 0,349 \\
\hline BI4 & 0,599 & 0,881 & 0,574 & 0,445 & 0,332 & 0,256 \\
\hline ENJ1 & 0,434 & 0,453 & 0,833 & 0,249 & 0,438 & 0,311 \\
\hline ENJ2 & 0,497 & 0,515 & 0,831 & 0,266 & 0,591 & 0,411 \\
\hline ENJ3 & 0,443 & 0,441 & 0,824 & 0,256 & 0,529 & 0,271 \\
\hline ENJ5 & 0,600 & 0,630 & 0,874 & 0,289 & 0,435 & 0,307 \\
\hline EXTR1 & 0,416 & 0,443 & 0,306 & 0,828 & 0,276 & 0,340 \\
\hline EXTR2 & 0,302 & 0,382 & 0,293 & 0,748 & 0,145 & 0,156 \\
\hline EXTR3 & 0,291 & 0,342 & 0,130 & 0,786 & 0,312 & 0,267 \\
\hline REP1 & 0,318 & 0,333 & 0,492 & 0,256 & 0,834 & 0,428 \\
\hline REP2 & 0,201 & 0,278 & 0,414 & 0,343 & 0,716 & 0,301 \\
\hline REP3 & 0,312 & 0,290 & 0,477 & 0,258 & 0,819 & 0,414 \\
\hline REP4 & 0,322 & 0,354 & 0,480 & 0,162 & 0,804 & 0,396 \\
\hline SUST1 & 0,434 & 0,289 & 0,339 & 0,307 & 0,433 & 0,854 \\
\hline SUST3 & 0,301 & 0,259 & 0,278 & 0,255 & 0,326 & 0,795 \\
\hline SUST4 & 0,387 & 0,181 & 0,296 & 0,250 & 0,393 & 0,808 \\
\hline SUST5 & 0,365 & 0,286 & 0,352 & 0,265 & 0,442 & 0,831 \\
\hline
\end{tabular}

